Research Paper

\title{
QRS-T-angle in Patients with ST-Segment Elevation Myocardial Infarction (STEMI) - a Comparison with Cardiac Magnetic Resonance Imaging
}

\author{
B. Zadeh ${ }^{1,2}$, J. M. Wambach ${ }^{1,2}$, M. Lambers ${ }^{1,2}$, K. Nassenstein 3 , C.J. Jensen ${ }^{1,2}$, O. Bruder ${ }^{1,2}{ }^{\bowtie}$ \\ 1. Department of Cardiology and Angiology, Contilia Heart and Vascular Center, Elisabeth-Krankenhaus Essen, Essen, Germany \\ 2. Ruhr University Bochum, Bochum, Germany \\ 3. Department of Diagnostic and Interventional Radiology and Neuroradiology, University Hospital Essen, Essen, Germany
}

$\triangle$ Corresponding author: Oliver Bruder, MD, Associate Professor of Medicine, Ruhr University Bochum, Department of Cardiology and Angiology, Contilia Heart and Vascular Center, Elisabeth-Krankenhaus Essen, Klara-Kopp-Weg 1, 45138 Essen, Germany. o.bruder@contilia.de; phone: +49 201 897 3200; fax: $\quad$ +49 201288525

(1) The author(s). This is an open access article distributed under the terms of the Creative Commons Attribution License (https://creativecommons.org/licenses/by/4.0/). See http://ivyspring.com/terms for full terms and conditions.

Received: 2020.01.27; Accepted: 2020.08.16; Published: 2020.08.25

\begin{abstract}
Background: The QRS-T angle from the surface EKG is a promising prognostic marker in patients with coronary artery disease. Cardiovascular magnetic resonance (CMR) imaging with late gadolinium enhancement (LGE) offers high resolution imaging of myocardial damage. We investigated the association of the QRS-T angle and the extent of myocardial damage as assessed by LGE in patients with acute ST-segment myocardial infarction (STEMI)

Methods: 169 patients with STEMI obtained a standardized digital 12-lead EKG on admission for the calculation of the QRS-T angle and underwent CMR imaging for analysis of infarct size by LGE within the first week. Patients were divided into groups: (1) abnormal QRS-T angle $\geq 90$ degree and (2) QRS-T angle $<90$ degree.

Results: Patients with a QRS-T angle of 90 degree or more had larger infarcts $(36.5 \pm 12.4$ vs. 13.3 \pm 9.5 ; $\mathrm{p}<0.001)$ and lower ejection fraction $(42.9 \pm 12.1 \%$ vs. $50.6 \pm 10.6 \% ; \mathrm{p}<0.001)$.

Conclusion: The extent of myocardial damage as measured by the gold standard LGE is associated with a larger QRS-T angle calculated from the surface EKG.
\end{abstract}

Key words: QRS-T angle, cardiovascular magnetic resonance, late gadolinium enhancement, ST-segment elevation myocardial infarction

\section{Introduction}

Early restoration of epicardial blood flow by percutaneous coronary intervention (PCI) or thrombolysis is the gold standard of ST-segment elevation myocardial infarction (STEMI) treatment [1, 2]. Risk stratification for recurrent myocardial infarction, heart failure and sudden cardiac death is key to treatment in the chronic phase of survived acute STEMI $[3,4]$.

Apart from clinical risk scores, biomarkers and imaging it would be extremely helpful to establish a simple parameter from the routine surface ECG for risk stratification in acute STEMI [5].

The QRS-T angle represents the spatial angle between the QRS loop (depolarization) and T loop (repolarization) [6]. A wide QRS-T angle $\left(\geq 90^{\circ}\right)$ seems to correlate with electrical instability and has been related to cardiac prognosis in several studies $[7,8]$.

Cardiovascular Magnetic Resonance (CMR) imaging following gadolinium contrast administration allows for high-resolution imaging of myocardial damage [9]. Late gadolinium enhancement (LGE) distribution patterns differentiate between ischemic (infarct) and non-ischemic (inflammation, fibrosis) myocardial pathology [10]. The extent of LGE is a strong and independent prognosticator in a variety of clinical settings [11, 12 , 
13]. In myocardial infarction CMR detects small subendocardial infarcts, and the transmural extent of LGE is related to left ventricular remodeling and recovery of function after revascularization [14].

This study aims to investigate the correlation of the QRS-T-angle on the 12-lead surface ECG and the extent of myocardial damage in patients with acute STEMI as assessed by late gadolinium enhancement CMR.

\section{Methods}

Consecutive patients admitted to the Department of Cardiology and Angiology at Elisabeth Hospital Essen from February 2015 until March 2017 with acute ST-segment elevation myocardial infarction (STEMI) were enrolled into the study. Patients obtained a standardized 12-lead digital ECG immediately on admission using a Schiller Cardiovit AT 102 plus ${ }^{\circledR}$. Computerized values of QRS and T-wave axis were given by the Schiller AT 102 plus ${ }^{\circledR}$ software. The frontal QRS-T-angle was calculated as the absolute difference between the frontal QRS- and frontal T-wave axes. With a value greater than $180^{\circ}$, $360^{\circ}$ were subtracted in order to give a continuous variable ranging from $0^{\circ}$ to $180^{\circ}$.

CMR imaging was performed on a 1.5 Tesla MR System (Magnetom Avanto ${ }^{\mathrm{TM}}$, Siemens Medical Solutions, Erlangen, Germany) not later than 7 days after admission. MR sequences were acquired using a phased-array receiver coil during end-inspiratory breathholding and in line with the recommendations of the Society of Cardiovascular Magnetic Resonance (SCMR) [15]. Contiguous short axis and three long axis cine images were acquired by using a steady-state free precession (SSFP) sequence. For Late Gadolinium Enhancement (LGE) imaging corresponding segmented inversion recovery gradient echo sequences (IR-GRE) short axis views were obtained every $10 \mathrm{~mm}$ (slice thickness $6 \mathrm{~mm}$ ) covering the entire left ventricle. LGE imaging was performed at least 10 minutes following the i.v. administration of 0.15 $\mathrm{mmol} / \mathrm{kgBW}$ gadoterate meglumine, adjusting the inversion time as described previously. In-plane resolution was typically $1.2 \times 1.8 \mathrm{~mm}$ [16].

Cine and LGE images were evaluated blinded to the clinical data by two experienced observers in consensus. LV function was analyzed by outlining epicardial and endocardial borders on the short axis SSFP sequences. Papillary muscles were excluded from analysis. Left ventricular volumes and ejection fraction were derived from contour summation. The extent of late gadolinium enhancement was assessed on long and short axis contrast images by using the 17-segment model of the LV. Typical expansion of LGE in patients with acute myocardial infarction
(STEMI) is defined as areas of high signal intensity progressing from the subendocardial myocardial layer. Areas with ischemic infarct scar were defined as enhanced with a signal intensity above at least five SD as compared to non-infarcted remote myocardium. The presence and transmurality of infarcts were scored using the 17-segment model and a 5-point scala per segment $(0=0 \%, 1=1-25 \%, 2=26-50 \%$, $3=51-75 \%, 4=76-100 \%)$. The total infarct size as expressed as percentage of LV mass was calculated as follows: sum of enhanced segments and their transmurality score (LGE score) divided by 17 segments multiplied by the maximum transmurality score [17].

Statistical analyses were performed with the Excel $^{\mathrm{TM}}$ software (Version 14.6.7, Microsoft ${ }^{\mathrm{TM}}$ ). Depending on the distribution continuous variables were expressed as means with standard deviation. Patients were divided into two groups (QRST-angle of 90 degree or larger vs smaller than 90 degree). The two-sample t-test was chosen to analyze differences between these two groups. Pearson correlation and one-sample t-test were applied to investigate the correlation of QRS-T angle and CMR parameters in each group. $\mathrm{P}$ values $<0.05$ were considered as statistically significant.

\section{Results}

From February 2015 to March 2017, 169 patients (125 male, 44 female, $60.8 \pm 11.1$ years) admitted for acute STEMI (121 anterior wall myocardial infarction, 43 inferior wall myocardial infarction) were enrolled.

Patients were divided into two groups: (1) patients with a frontal QRS-T angle of 90 degree or more $(n=54)$, (2) patients with a frontal QRS-T angle of less than 90 degree $(n=114)$ (Table 1$)$.

Mean QRS-T angle in group 1 and group 2 was $125.8 \pm 38.3$ and $33 \pm 21.1$ respectively. Patients with a QRS-T angle of 90 or more were older $(63.7 \pm 11.2$ vs. $59.4 \pm 10.9 ; \mathrm{p}<0.05)$ and the heart rate on admission EKG was higher $(85 \pm 17 \mathrm{bpm}$ vs. $77 \pm 17 \mathrm{bpm}$; $\mathrm{p}<0.05)$. There was no difference between the two groups with regards to maximum creatinkinase (group 1: $1452 \pm 1778.2 \mathrm{U} / 1$ vs. group 2: $1451 \pm 1741.1 \mathrm{U} / \mathrm{l} ; \mathrm{p}=0.99$ ) and maximum troponine (group $1: 3.0 \pm 3.1 \mathrm{ng} / \mathrm{ml}$ vs. group 2: $3.4 \pm 4.0 \mathrm{ng} / \mathrm{l} ; \mathrm{p}=0.44)$.

On CMR imaging (Figure 1, Table 1) ejection fraction was lower with a QRS-T angle of 90 degree or more $(42.9 \pm 12.1 \%$ vs. $50.6 \pm 10.6 \%$; p<0.001). 50 of 114 patients in group 2 had a normal left ventricular ejection fraction, whereas ejection fraction was $45 \%$ or less in 34 of 54 patients in group 1. The extent of myocardial necrosis as represented by percent late gadolinium enhancement of LV mass (LVM) was significantly $(\mathrm{p}<0.001)$ higher $(36.5 \pm 12.4)$ in group 1 
with a QRS-T angle of 90 or more as compared to group 2 (13.3 \pm 9.5$)$. In addition, there is a moderate correlation of LGE percent LVM and the QRS-T angle (Figure 2, r=0.65936).

Table 1: Clinical and CMR Parameter for QRS-Tangle $\geq 90$ vs. QRS-T angle $<90$

\begin{tabular}{llll}
\hline Parameters & $\begin{array}{l}\text { QRS-T } \geq \mathbf{9 0}(\mathbf{n}= \\
\mathbf{5 4})\end{array}$ & $\begin{array}{l}\text { QRS-T }<\mathbf{9 0}(\mathbf{n}= \\
\mathbf{1 1 4})\end{array}$ & $\begin{array}{l}\mathbf{p}- \\
\text { value }\end{array}$ \\
\hline $\begin{array}{l}\text { Demographic Data } \\
\text { gender }(\mathrm{w} / \mathrm{m}) \text { in \% }\end{array}$ & $26 / 74$ & $27 / 73$ & \\
QRS-T angle (degree) & $125.8 \pm 38.3$ & $33.0 \pm 21.1$ & $<.957$ \\
age at infarction (years) & $63.7 \pm 1.2$ & $59.4 \pm 10.9$ & $\mathbf{0 . 0 1 9}$ \\
heart rate (bpm) & $85.2 \pm 17.3$ & $76.6 \pm 17.2$ & $\mathbf{0 . 0 0 3}$ \\
CMR Data & & & \\
ejection fraction, EF $(\%)$ & $42.9 \pm 12.1$ & $50.6 \pm 10.6$ & $<0.001$ \\
ESV (ml) & $96 \pm 42$ & $80 \pm 32.1$ & 0.089 \\
\hline
\end{tabular}

\begin{tabular}{llll}
\hline Parameters & $\begin{array}{l}\text { QRS-T } \geq \mathbf{9 0}(\mathbf{n}= \\
\mathbf{5 4})\end{array}$ & $\begin{array}{l}\text { QRS-T }<\mathbf{9 0}(\mathbf{n}= \\
\mathbf{1 1 4})\end{array}$ & $\begin{array}{l}\mathbf{p}- \\
\text { value }\end{array}$ \\
\hline EDV $(\mathrm{ml})$ & $161 \pm 46$ & $148 \pm 39$ & $\mathbf{0 . 0 0 3}$ \\
pericardial effusion $(\mathrm{mm})$ & $3.1 \pm 4.1$ & $1.0 \pm 2.1$ & $\mathbf{0 . 0 3 4}$ \\
LGE in \% LV mass & $36.5 \pm 12.4$ & $13.3 \pm 9.5$ & $<0.001$ \\
no reflow zone (number of & 11 & 14 & 0.121
\end{tabular}
patients)

Clinical Data

$\begin{array}{llll}\text { ck maximum }(\mathrm{U} / \mathrm{l}) & 1452 \pm 1778.2 & 1451 \pm 1741.1 & 0.998\end{array}$

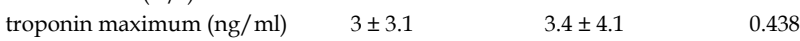

$\begin{array}{llll}\text { Cardiovascular risk factors } & & 65 & 0.004\end{array}$

$\begin{array}{llll}\text { arterial hypertension } & 43 & 65 & \mathbf{0 . 0 0 4} \\ \text { family disposition } & 14 & 31 & 0.860\end{array}$

hyperlipidaemi

diabetes mellitus

obesity

smoking

LGE late gadolinium enhancement, LV left ventricular, ESV end-systolic volume, EDV end-diastolic volume

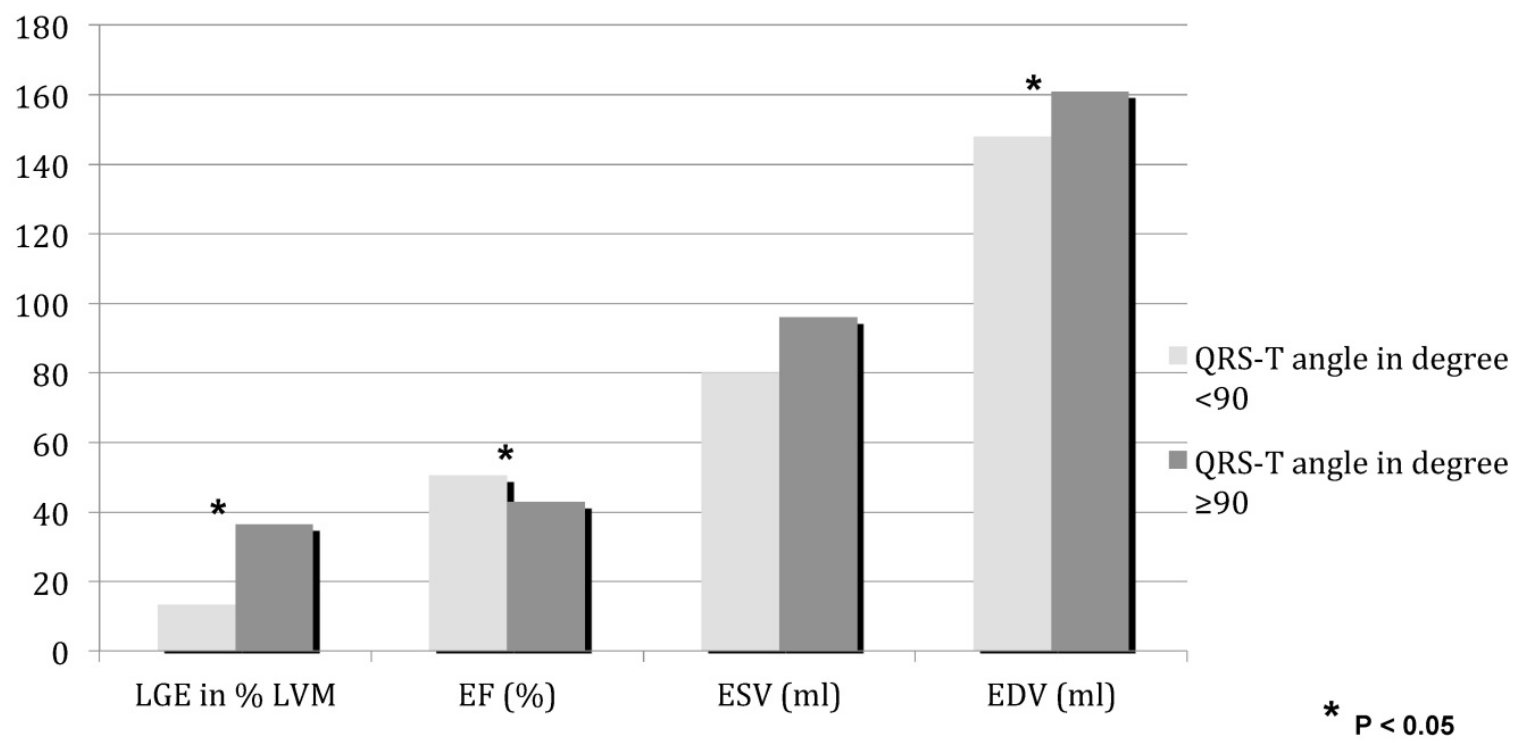

Figure 1: CMR parameters for QRS-T angle $\geq 90$ (dark) and QRS-T angle $<90$ (bright). LGE late gadolinium enhancement, LVM left ventricular mass, EF ejection fraction, ESV end-systolic volume,EDV end-diastolic volume

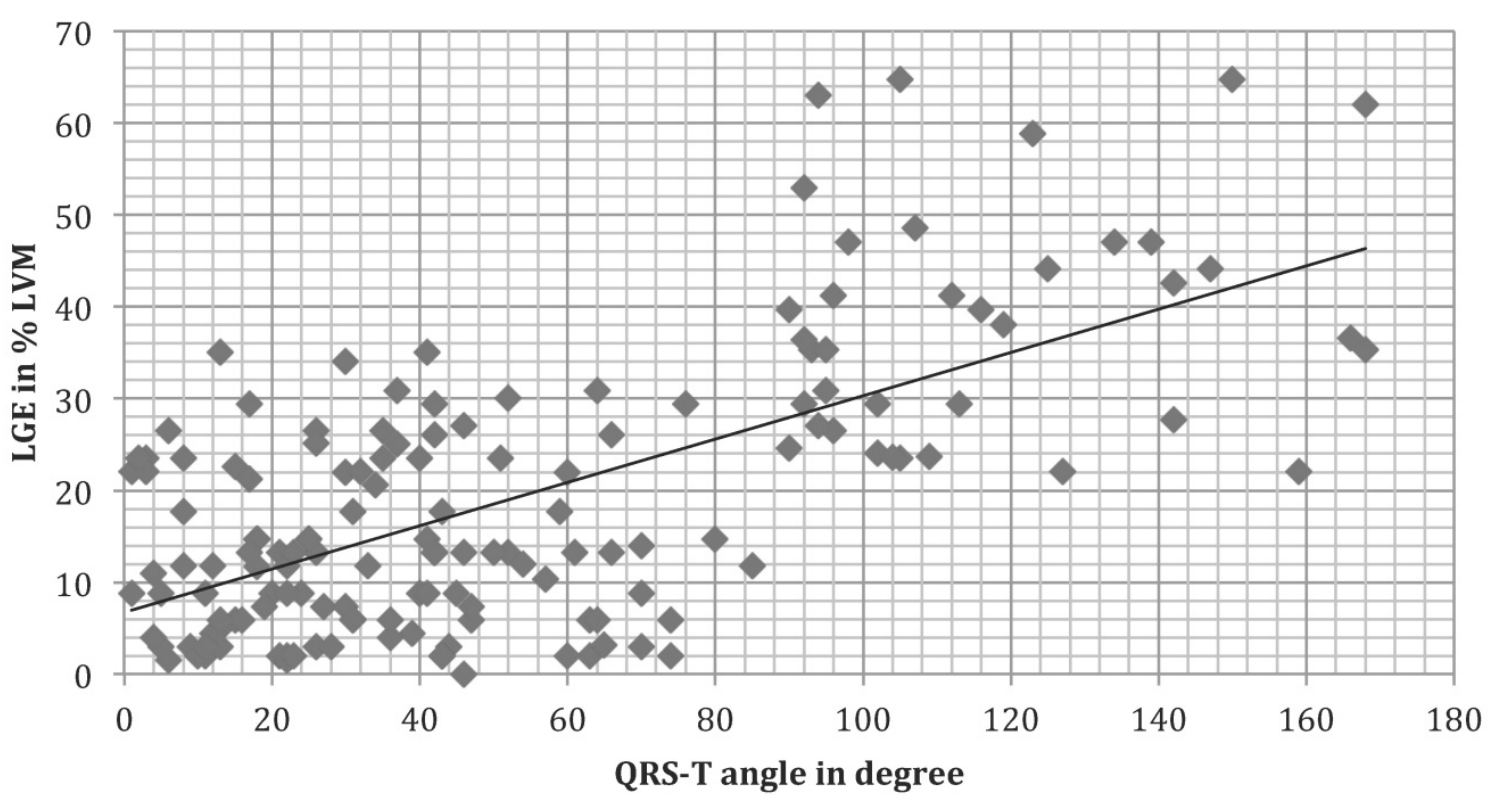

Figure 2: LGE score versus QRS-T angle. Pearson correlation coefficient was $r=0.65936$. LGE late gadolinium enhancement, LVM left ventricular mass 


\section{Discussion}

Our study shows that in a population of STEMI patients QRS-T angle on the surface EKG correlates with infarct size on contrast-enhanced CMR.

Previously several studies have been able to demonstrate the prognostic value of QRS-T angle in different patient populations. In a general Rotterdam population of 6,134 patients Kors et al. found an odds ratio of 5.6 for sudden cardiac death, which was superior to standard EKG indicators of adverse events such as ST depression and T wave inversion [18]. Borleffs et al. detected that in a group of 412 ICD carriers a wide QRS-T angle was highly predictive of adequate ICD discharge and ventricular tachycardia [19]. In 2705 consecutive patients with suspected NSTEMI QRS-T angle adds diagnostic accuracy as compared to standard EKG criteria of ischemia alone and predicts cardiac mortality [20]. A frontal QRS-T angle of more than 90 degrees in 467 STEMI survivors with reduced ejection fraction demonstrated a strong and independent prognostic value [21].

Several ECG signs have been evaluated against distribution, transmurality and extent of late gadolinium enhancement on CMR following gadolinium contrast administration: In acute STEMI the resolution of ST-segment elevation, a marker of successful reperfusion therapy, relates to LGE and prognosis [22]. A fragmentation of the QRS complex, however, does not predict late gadolinium enhancement in acute myocardial infarction [23]. And ST-segment elevation in the right precordial leads indicates right ventricular involvement in patients with inferior wall myocardial infarction only in larger right ventricular infarcts if LGE of the right ventricle serves as reference standard [24]. In chronic infarction, LGE identifies infarct scars in patients with clinically silent myocardial infarction that do no exhibit significant $Q$ waves on the surface EKG $[25$, 26]. Moon et al. found that in a group of 100 consecutive patients with chronic infarction the presence of $Q$ waves was related to infarct size more than to infarct transmurality on late gadolinium enhancement CMR [27].

The concept of validating EKG parameters by scar (LGE) rather than left ventricular morphology, hypertrophy and function has also been investigated in the setting of non-ischemic cardiomyopathies: In hypertrophic cardiomyopathy late gadolinium enhancement is a strong and independent prognosticator of cardiac death [28, 29]. Delcrè et al. reported a strong correlation of an EKG score including 9 independent markers and late gadolinium enhancement size in 257 patients with hypertrophic cardiomyopathy [30].
For a prognostic EKG marker that represents myocardial depolarization and repolarization in the way the QRS-T angle does, the correlation to myocardial pathology on CMR is particularly useful. To the best of our knowledge, this is the first study that relates QRS-T angle in patients with acute myocardial infarction (STEMI) to CMR function and scar.

Several non-invasive parameters that could help risk stratify patients with acute myocardial infarction are used in clinical routine. These include left ventricular ejection fraction, left ventricular volumes, several ECG parameters and detection of myocardial necrosis by contrast-enhanced cardiovascular magnetic resonance imaging. The ultimate goal in risk stratification is to identify patients who will suffer from sudden cardiac death and who need further protection, e.g. the implantation of cardioverterdefibrillator (ICD). However, up till now no single parameter has been successful to fulfill the task. In fact, due to the complex pathophysiology of ventricular arrhythmias a combination of non-invasive parameters might improve the prediction of ventricular arrhythmias in post acute MI patients. The QRS-T angle in patients with acute MI can be ubiquitously assessed and might add to clinical risk stratification in the future.

In conclusion, a QRS-T angle of 90 degrees or more is associated with lower ejection fraction and greater infarct size as assessed by contrast-enhanced CMR. Prospective studies in a larger STEMI patient population with clinical follow-up should be carried out in order to further investigate the prognostic impact of the QRS-T angle and its potential implication on clinical workflow.

\section{Competing Interests}

The authors have declared that no competing interest exists.

\section{References}

1. Keeley EC, Boura JA, Grines CL. Primary angioplasty versus intravenous thrombolytic therapy for acute myocardial infarction: a quantitative review of 23 randomised trials. Lancet. 2003;361(9351):13-20.

2. Bednár F, Widimský P, Krupicka J, et al. Interhospital transport for primary angioplasty improves the long-term outcome of acute myocardial infarction compared with immediate thrombolysis in the nearest hospital (one-year follow-up of the PRAGUE-1 study). Can J Cardiol. 2003;19(10):1133-1137.

3. St John Sutton M, Pfeffer MA, Plappert T, et al. Quantitative two-dimensional echocardiographic measurements are major predictors of adverse cardiovascular events after acute myocardial infarction. The protective effects of captopril. Circulation. 1994;89(1):68-75.

4. Moss AJ, Zareba W, Hall WJ, et al. Prophylactic implantation of a defibrillator in patients with myocardial infarction and reduced ejection fraction. $N$ Engl J Med. 2002;346(12):877-883.

5. Fox KA, Dabbous OH, Goldberg RJ, et al. Prediction of risk of death and myocardial infarction in the six months after presentation with acute coronary syndrome: prospective multinational observational study (GRACE). BMJ. 2006;333(7578):1091.

6. Oehler A, Feldman T, Henrikson CA, et al. QRS-T angle: a review. Ann Noninvasive Electrocardiol. 2014;19(6):534-542.

7. Zhang ZM, Prineas RJ, Case D, et al. ARIC Research Group. Comparison of the prognostic significance of the electrocardiographic QRS/T angles in 
predicting incident coronary heart disease and total mortality (from the atherosclerosis risk in communities study). Am J Cardiol. 2007;100(5):844-849.

8. Aro AL, Huikuri HV, Tikkanen JT, et al. QRS-T angle as a predictor of sudden cardiac death in a middle-aged general population. Europace. 2012;14(6):872876.

9. Wagner A, Mahrholdt H, Holly TA, et al. Contrast-enhanced MRI and routine single photon emission computed tomography (SPECT) perfusion imaging for detection of subendocardial myocardial infarcts: an imaging study. Lancet. 2003;361(9355):374-379.

10. Mahrholdt H, Wagner A, Judd RM, et al. Delayed enhancement cardiovascular magnetic resonance assessment of non-ischaemic cardiomyopathies. Eur Heart J. 2005;26(15):1461-1474.

11. Chan RH, Maron BJ, Olivotto I, et al. Prognostic value of quantitative contrast-enhanced cardiovascular magnetic resonance for the evaluation of sudden death risk in patients with hypertrophic cardiomyopathy. Circulation. 2014;130(6):484-495.

12. Grün S, Schumm J, Greulich $S$, et al. Long-term follow-up of biopsy-proven viral myocarditis: predictors of mortality and incomplete recovery. J Am Coll Cardiol. 2012;59(18):1604-1615.

13. Klem I, Shah DJ, White RD, et al. Prognostic value of routine cardiac magnetic resonance assessment of left ventricular ejection fraction and myocardial damage: an international, multicenter study. Circ Cardiovasc Imaging. 2011;4(6):610-619.

14. Kim RJ, Wu E, Rafael $\mathrm{A}$, et al. The use of contrast-enhanced magnetic resonance imaging to identify reversible myocardial dysfunction. $N$ Engl J Med. 2000;343(20):1445-1453.

15. Kramer CM, Barkhausen J, Flamm SD, et al. Society for Cardiovascular Magnetic Resonance Board of Trustees Task Force on Standardized Protocols. Standardized cardiovascular magnetic resonance (CMR) protocols 2013 update. J Cardiovasc Magn Reson. 2013;15(1):91.

16. Jensen CJ, Eberle HC, Nassenstein $\mathrm{K}$. et al. Impact of hyperglycemia at admission in patients with acute ST-segment elevation myocardial infarction as assessed by contrast-enhanced MRI. Clin Res Cardiol. 2011;100(8):649-59.

17. Klem I, Heiber E, van Asschee L, Parker MA, Kim HW, Grizzard JD, Arheden $\mathrm{H}, \mathrm{Kim}$ RJ. Sources of variability in quantification of cardiovascular magentic resonance infarct size - reproducibility among three core laboratories. J Cardiovasc Magn Reson. 2017; 19:62

18. Kors JA, Kardys I, van der Meer IM, et al. Spatial QRS-T angle as a risk indicator of cardiac death in an elderly population. J Electrocardiol. 2003; 36(Suppl):113-4.

19. Borleffs CJ, Scherptong RW, Man SC, et al. Predicting ventricular arrhythmias in patients with ischemic heart disease: clinical application of the ECG-derived QRS-T angle. Circ Arrhythm Electrophysiol. 2009;2(5):548-554

20. Strebel I, Twerenbold R, Wussler D, et al. Incremental diagnostic and prognostic value of the QRS-T angle, a 12-lead ECG marker quantifying heterogeneity of depolarization and repolarization, in patients with suspected non-ST-elevation myocardial infarction. Int J Cardiol. 2019;277:8-15.

21. Raposeiras-Roubín S, Virgós-Lamela A, Bouzas-Cruz N, et al. Usefulness of the QRS-T angle to improve long-term risk stratification of patients with acute myocardial infarction and depressed left ventricular ejection fraction. Am J Cardiol. 2014;113(8):1312-1319.

22. Nijveldt R, van der Vleuten PA, Hirsch A, et al. Early electrocardiographic findings and MR imaging-verified microvascular injury and myocardial infarct size. JACC Cardiovasc Imaging. 2009; 2(10):1187-94.

23. Ahn MS1, Kim JB, Yoo BS, et al. Fragmented ORS complexes are not hallmarks of myocardial injury as detected by cardiac magnetic resonance imaging in patients with acute myocardial infarction. Int J Cardiol. 2013;168(3):2008-13

24. Jensen CJ, Jochims M, Hunold P, et al. Right ventricular involvement in acute left ventricular myocardial infarction: prognostic implications of MRI findings. AJR Am J Roentgenol. 2010;194(3):592-598.

25. Kwong RY, Sattar $\mathrm{H}, \mathrm{Wu} \mathrm{H}$, et al. Incidence and prognostic implication of unrecognized myocardial scar characterized by cardiac magnetic resonance in diabetic patients without clinical evidence of myocardial infarction. Circulation. 2008;118(10):1011-1020.

26. Acharya $\mathrm{T}$, Aspelund $\mathrm{T}$, Jonasson $\mathrm{TF}$, et al. Association of Unrecognized Myocardial Infarction With Long-term Outcomes in Community-Dwelling Older Adults: The ICELAND MI Study. JAMA Cardiol. 2018;3(11):1101-1106.

27. Moon J, De Arenaza D, Elkington A, et al. The Pathologic Basis of Q-Wave and Non-Q-Wave Myocardial Infarction A Cardiovascular Magnetic Resonance Study. Journal of the American College of Cardiology 2004; 0735-1097.

28. Bruder O, Wagner A, Jensen CJ, et al. Myocardial scar visualized by cardiovascular magnetic resonance imaging predicts major adverse events in patients with hypertrophic cardiomyopathy. J Am Coll Cardiol. 2010;56(11):875-887.

29. O'Hanlon R, Grasso A, Roughton M, et al. Prognostic significance of myocardial fibrosis in hypertrophic cardiomyopathy. J Am Coll Cardiol. 2010;56(11):867-874.

30. Delcrè SD, Di Donna $\mathrm{P}$, Leuzzi S, et al. Relationship of ECG findings to phenotypic expression in patients with hypertrophic cardiomyopathy: a cardiac magnetic resonance study. Int J Cardiol 2013; 10;167(3):1038-45. 